\title{
Transient tracer dynamics in a lattice-automaton model of bioturbation
}

\author{
by Daniel C. Reed $^{1,2}$, Bernard P. Boudreau ${ }^{1}$ and Katherine Huang ${ }^{1}$
}

\begin{abstract}
Mixing of sediments by benthic fauna represents a dominant transport process of solids in the majority of surficial marine deposits. Short-term mixing rates are often determined by fitting a transient, diffusive model of bioturbation to vertical profiles of introduced particles (e.g., luminophores). Previous field studies adopting this approach have noted that mixing intensity decreases with time and the nature of mixing progresses from advective or nonlocal transport to diffusive mixing. These observations have been attributed to "age-dependent" mixing.

The present study employs a lattice-automaton model to investigate the short-term behavior of conservative transient tracers. Simulations demonstrate that despite constant, indiscriminate mixing of particles, mixing intensity, as quantified by the diffusion model, appears as a function of time due to the model being invalid on short time scales. Failure of the model, however, is not apparent from tracer profiles. Furthermore, the errors incurred by misapplying the biodiffusion model can be considerable with estimates of mixing intensity reaching up to $3000 \%$ of the actual value. The transition from advective to diffusive mixing is also demonstrated and found to be the product of a boundary effect. Results presented here suggest that, on average, at least nine mixing events are required before the biodiffusion model becomes an appropriate description of bioturbation. Thus, providing the time scale of the tracer is sufficiently large relative to the time scale of mixing, the model provides a reasonable description of bioturbation.
\end{abstract}

\section{Introduction}

Marine sediments are host to an impressive variety of organisms. Routine activities of such organisms fundamentally alter the physical structure and geochemistry of sediments by mixing sediment particles, a process termed bioturbation (Rhoads, 1974). As bioturbation is frequently the dominant particle transport process in surficial sediment deposits, the manner in which organisms displace particles is critical to the distribution of chemical species (e.g., organic matter) and, consequently, to chemical cycling (Aller, 1982). Moreover, biogenic mixing regulates fluxes across the sediment-water interface, thus, playing an important role in the exchange of nutrients, contaminants, and other relevant species between sediments

1. Department of Oceanography, Dalhousie University, 1355 Oxford Street, Halifax, Nova Scotia, Canada, B3H $4 \mathrm{~J} 1$.

2. Present address: Department of Geochemistry, Faculty of Earth Sciences, Utrecht University, 3508 TA Utrecht, The Netherlands. email: D.Reed@geo.uu.nl. 
and overlying waters (Aller, 1988; Bosworth and Thibodeaux, 1990). Bioturbation is also significant from a paleoceanographic standpoint, as mixing impedes the interpretation of the sedimentary record by smearing the deposition input signal, an effect which must be accounted for during analysis (Berger and Heath, 1968).

Owing to the significant influence of bioturbation on numerous sedimentary processes and properties, a quantitative description of biological mixing is needed. Various models of bioturbation have been developed with this in mind (e.g., Goreau, 1977; Boudreau and Imboden, 1987; François et al., 1997; Shull, 2001), although none have seen such widespread application as the biodiffusion model. This model, introduced by Goldberg and Koide (1962) and popularized by Guinasso and Schink (1975), features biological transport as a diffusive flux with the intensity of mixing quantified by the biodiffusion coefficient, $D_{b}$. The magnitude of $D_{b}$ is usually determined by fitting model solutions to tracer profiles. Both time-dependent and steady-state particle-bound radioisotope tracers (e.g., ${ }^{137} \mathrm{Cs},{ }^{210} \mathrm{~Pb}$ ) are used (e.g., Cochran, 1985; DeMaster et al., 1985), as well as introduced particles, such as luminophores (e.g., Solan et al., 2004) and glass beads (e.g., Wheatcroft, 1992). Traditionally, only the vertical dimension is considered, as chemical gradients tend to be steepest in this direction (Berner, 1980); consequently, lateral mixing has only been investigated to a limited degree (Wheatcroft, 1991).

The ubiquitous application of the biodiffusion model is largely due to its simple mathematical form, three decades of apparent success, and the ability to reduce a myriad of different animal-sediment interactions to a single parameter. Indeed, by characterizing bioturbation as a diffusive flux, diagenetic models can account for biological transport in a single, straightforward term without concern for the specifics of community composition or mixing mechanisms at work. However, the diffusion analogy has limitations. Specifically, the model is only appropriate when certain criteria are fulfilled regarding the frequency, magnitude, and symmetry of particle displacements (Boudreau, 1986a; Meysman et al., 2003). Recent studies suggest these model assumptions are invalid on short time-scales, like those of short-lived radioisotope tracers (Meysman et al., 2003; Reed et al., 2006). Failure of the model, however, is not necessarily apparent from tracer profiles, allowing the model to be unknowingly misapplied. Reed et al. (2006) have demonstrated that misuse of the model can fabricate a relationship between $D_{b}$ and the half-life of the steady-state radionuclide tracer used. When the necessary conditions of the biodiffusion model are not fulfilled, the intensity of mixing appears to be a function of tracer half-life, as observed in nature (Smith et al., 1993, 1997; Turnewitsch et al., 2000). In this case, mixing coefficients derived from short-lived radionuclides significantly overestimate mixing due to model failure and, as a consequence, caution must be exercised when using the biodiffusion model to interpret data.

In sediments, organic matter is of paramount importance from both a geochemical and biological perspective. Organics degrade relatively quickly providing motivation to study diagenetic processes on short time scales. Short-term mixing rates are typically determined by modeling either introduced particles or short-lived radioisotope tracers. Previous studies 
using transient tracers have shown $D_{b}$ as a decreasing function of time with the nature of bioturbation shifting from initial bioadvective or nonlocal transport to diffusive mixing (Fornes et al., 2001; Smith et al., 2001). Such observations have been attributed to so-called "age-dependent" mixing, whereby particles are mixed preferentially depending on how recently they were deposited.

To investigate the short-term behavior of transient tracers, the current study employs a lattice-automaton model of bioturbation. Simulations presented here demonstrate that the temporal trend in $D_{b}$ observed in nature occurs inherently, even when mixing is constant and indiscriminate. Furthermore, the apparent shift in mixing behavior is shown to be intrinsic to bioturbation in the absence of particle-selectivity.

\section{The Lattice-Automaton Bioturbation Simulator}

The Lattice-Automaton Bioturbation Simulator (LABS) is a computer model comprising a two-dimensional matrix that represents a vertical plane in a sediment, populated by automatons representing benthic fauna. Each of the cells in the lattice are designated as either porewater, particle, or organism. An automaton consists of a group of cells that moves in unison through the sediment-water matrix. The behavior of the automatons is governed by both stochastic and deterministic rules prescribed to replicate the activities of actual fauna, such as burrowing through the sediment-water matrix, feeding on particles, and defecating previously ingested particles. Several types of automaton currently exist in the model: funnel-feeders (e.g., Arenicola marina), burrow-and-fill mixers (e.g., fiddler crabs), subsurface deposit-feeding worms, and head-down deposit-feeding worms. In the simulations presented here, the model is populated with mobile subsurface deposit-feeding worms. The burrowing rules for these worms are based on the fracture mechanism proposed by Dorgan et al. (2005), whereby the sediment is cracked open by the organism in the same manner as a bubble propagates through a sediment (Johnson et al., 2002). Automatons clear the particles in their path from the center of their body outwards, leaving a porewater-filled burrow with a compacted wall in their wake. These compacted regions decay in time (i.e., the particles fall apart randomly). As the automatons burrow through the lattice they ingest particles, which are carried in their gut until they are egested elsewhere. Owing to the random nature of burrowing and indiscriminate choice of particles, there are no distinct regions for feeding or defecation. At the lateral boundaries, both particles and automatons wrap from one side of the matrix to the other, ensuring conservation of mass. The user provides parameters relating to their behavior, such as burrowing and ingestion rates, as well as other parameters for defining the system, for instance, sedimentation rate, matrix dimensions and mixed layer depth. In LABS, the mixed-layer depth defines the position of the lower boundary, beyond which the automatons cannot venture.

LABS was originally written in FORTRAN (Choi et al., 2002), but has recently been recast in an object-oriented design and coded in $\mathrm{C}++$. The motivation for developing such a model is twofold: (1) The explicit inclusion of organisms in the model allows crucial, 
mechanistic links to be established between sediment mixing and faunal activities, parameters, and behavior (Boudreau et al., 2001); (2) Mixing can be observed simultaneously on both the level of individual particles and distributions of particles (i.e., tracer profiles), allowing information furnished by inverse models to be analysed and corroborated (Reed et al., 2006). The latter is the focus of this paper. Specifically, the time-dependent biodiffusion model is examined by simulating luminophore experiments in LABS. Particles designated as luminophores are added to the model, allowing profiles to be obtained by taking lateral slices of the sediment matrix and counting the number of luminophore particles in each slice, in the same vein as field and laboratory experiments. Fitting the biodiffusion model to such profiles furnishes mixing parameters, which can be compared to mixing parameters derived solely from particle displacement statistics. In this way, the tracer-derived values can be verified. The result is an insight into how the time-dependent biodiffusion model perceives mixing on short time scales.

\section{Quantifying bioturbation}

\section{a. The biodiffusion model}

The biodiffusion model is the canonical approach to describing the distribution of both steady-state and time-dependent tracers subject to biogenic mixing. When considering introduced particles, the initial distribution is represented as a pulse at the sediment-water interface with subsequent dispersion of the tracer described by Fick's laws of diffusion. The diffusion analogy, however, cannot account for movement of the profile maximum from the sediment-water interface. In such a case, an advective term is usually included in the model, termed bioadvection (e.g., Gerino et al., 1998). Traditionally, bioadvective transport is attributed to "conveyor-belt" species (Rhoads, 1974), who ingest sediment at depth and subsequently defecate this material on the sediment-water interface (e.g., Fisher et al., 1980; Boudreau, 1986b; Rice, 1986). Alternatively, tracer particles falling into existing burrows provides another advective mechanism (Tedesco and Wanless, 1991; Gerino et al., 1994).

The time-dependent biodiffusion model for a conservative tracer is,

$$
\frac{\partial C}{\partial t}=D_{b}^{t} \frac{\partial^{2} C}{\partial x^{2}}-v_{b}^{t} \frac{\partial C}{\partial x}
$$

where $C$ is the tracer activity, $D_{b}^{t}$ is the constant biodiffusion coefficient, $v_{b}^{t}$ is the constant bioadvection coefficient, $x$ is distance from the sediment-water interface, and $t$ is time. The superscript $t$ denotes that the transport parameters are derived from tracer profiles. Eq. 1 assumes constant porosity and mixing parameters, as well as negligible sedimentation. Typical boundary conditions are,

$$
\begin{gathered}
D_{b}^{t} \frac{\partial C}{\partial x}-v_{b}^{t} C=0, \text { at } x=0 \\
\frac{\partial C}{\partial x}=0, \text { as } x \rightarrow \infty
\end{gathered}
$$


with the Dirac delta function, $\delta(x)$, as the initial condition. Eq. 2 represents no flux at the sediment-water interface, meaning no tracer is lost to the water column nor introduced after the initial pulse, while equation 3 ensures a finite amount of tracer at depth. Solving Eq. 1 with these initial and boundary conditions furnishes,

$$
C(x, t)=\frac{1}{\sqrt{\pi D_{b}^{t} t}} e^{\left(\frac{-\left(x-v_{b}^{t} t\right)^{2}}{4 D_{b}^{t} t}\right)}-\frac{v_{b}^{t}}{2 D_{b}^{t}} e^{\left(\frac{v_{b}^{t}}{D_{b}^{t}} x\right)} \operatorname{erfc}\left(\frac{x+v_{b}^{t} t}{\sqrt{4 D_{b}^{t} t}}\right)
$$

(Carslaw and Jaeger, 1965; Officer and Lynch, 1982). When fit to tracer profiles, this equation provides values for $D_{b}^{t}$ and $v_{b}^{t}$.

\section{b. Particle-tracking in LABS}

An alternative approach to quantifying mixing in LABS is by tracking the displacements of individual particles. The statistics of particle movements can be used to construct a random walk model of particle transport, a description that is tracer-independent. Such a model treats particle displacements as stochastic events, whereby particles "jump" from one location in the sediment to another. The concept of modeling bioturbation as a random walk process was first introduced by Boudreau (1989) and Wheatcroft et al. (1990). In their random walk model, Wheatcroft et al. (1990) assumed the magnitude and frequency of particle displacements were represented by mean values, estimated from field and laboratory observations. Particle transport was also assumed to be isotropic and, consequently, no advective drift appeared in the model. In nature, particle displacements often exhibit a directional bias and are variable in magnitude and frequency, due to the variety of organisms present and mixing mechanisms at work. To account for this explicitly, the approach adopted here is the same as Reed et al. (2006), which allows for variability in the frequency, magnitude and directionality of particle displacements. For this method, two probability density functions (PDF) are required: the first describes the amount of time a particle is stationary, waiting to be displaced (i.e., the rest period); the second describes the magnitude and direction of transport (i.e., the step length). These distributions can be combined into a stochastic model of the behavior of an individual particle, which may then be extrapolated into a deterministic description of the entire population of particles (Reed et al., 2006). In the long time limit, providing the rest period PDF has a finite mean and the step length PDF has a finite mean and variance (unlike a Lévy distribution, for example), the random walk model tends to the diffusion-advection equation (Hughes, 1995). That is, after a sufficient number of mixing events the biodiffusion model is achieved. In this case, the diffusion coefficient, termed the particle-tracking biodiffusion coefficient $\left(D_{b}^{p}\right)$, is of the form,

$$
D_{b}^{p}=\frac{\sigma^{2}}{2 \tau}
$$

where $\sigma^{2}$ is the variance of the step length PDF and $\tau$ is the mean of the rest period PDF. The characteristic spatial and temporal scales of mixing are, therefore, $\sigma$ and $\tau$, respectively. 
Any directional bias appears as an advective drift term with the coefficient, similarly termed the particle-tracking bioadvection coefficient $\left(v_{b}^{p}\right)$,

$$
v_{b}^{p}=\frac{\mu}{\tau}
$$

where $\mu$ is the mean of the step length PDF. A bias in the direction of particle transport can arise from many different sources, for example, infilling of burrows (Benninger et al., 1979), caching feeding strategies (Jumars et al., 1990), "conveyor-belt" mixing (Fisher et al., 1980), among others. By recording all particle displacements in LABS, step length and rest period distributions can be constructed and used to calculate tracer-independent measures of mixing. This provides the opportunity to validate tracer-derived mixing measures. In LABS, the step length and rest period PDFs are a function of depth and, as a result, the particletracking transport parameters are also depth-dependent, as seen in Section 4a. While, in contrast, Section $4 \mathrm{~d}$ shows that particle mixing in LABS is invariable in time. Further details on the particle-tracking approach can be found in Reed et al. (2006).

\section{Model output and discussion}

An initial LABS run was undertaken with a population of mobile subsurface depositfeeding worms over a period of 5 years. The automatons are comparable to Capitella spp. in terms of size $(0.2 \times 2 \mathrm{~cm}$; Grassle and Grassle, 1974), gut-to-body ratio (0.18; Penry and Jumars, 1990) and ingestion rate (50 $\left.\mathrm{mg} \mathrm{sed}^{-1}\right)$. This feeding rate is quite modest,

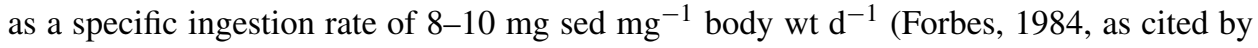
Lopez and Levinton, 1987) coupled with body weights of 3-12 mg (Grassle and Grassle, 1976) furnish a range of rates of $24-120 \mathrm{mg} \mathrm{d}^{-1}$, as smallest organisms correspond to lowest ingestion rates and vice versa (Méndez et al., 2001). The faunal density was set at 5000 individuals $\mathrm{m}^{-2}$ with a burrowing rate of $20 \mathrm{~cm} \mathrm{~d}^{-1}$ (cf. Caron et al., 1996). The mixed-layer depth was assumed as the global average of $10 \mathrm{~cm}$ (Boudreau, 1994) with a lateral domain of $50 \mathrm{~cm}$ and there was no sedimentation. At the outset, a $5 \mathrm{~mm}$ layer of luminophores was deposited on the sediment-water interface and the model was initialized. Tracer profiles were output every 10 days for the first 1000 days and the model continued to run up until 5 years, at which point particle-tracking data were output to ensure enough particle-movements to define the PDFs.

\section{a. Temporal evolution of tracer profiles}

Figure 1 shows the tracer distribution versus time, from day 10 to day 1000. While mixing has dispersed the tracer somewhat by day 10 , the initial pulse is still evident at the sediment-water interface. In the early stages of the simulation the tracer is subducted and then, subsequently, dissipated by mixing. In situ studies have observed similar behavior, whereby bioturbation switches from initial advective or nonlocal transport to diffusive mixing (Fornes et al., 2001; Smith et al., 2001). Paradoxically, this transition is not due to a change in mixing mechanisms, but rather how mixing mechanisms manifest themselves. 


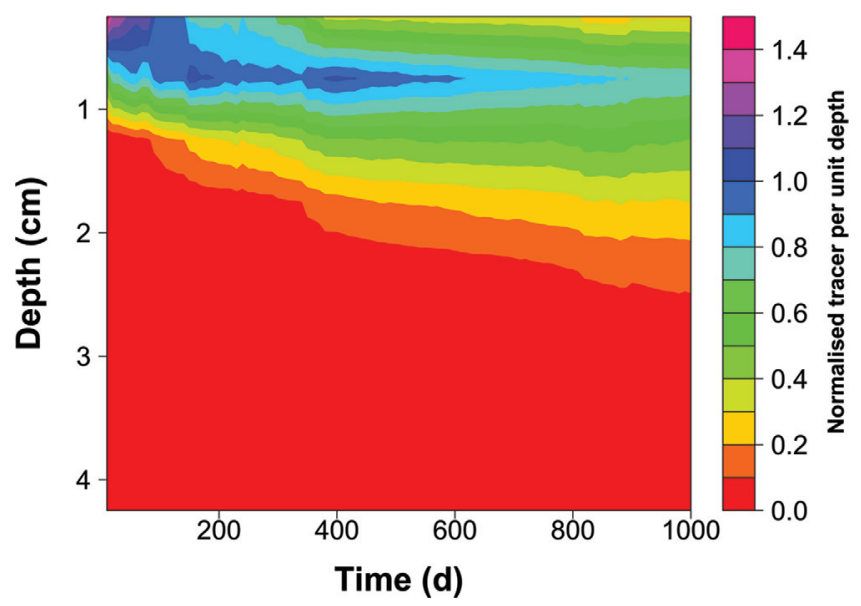

Figure 1. The depth distribution of a conservative tracer from day 10 to day 1000 .

In LABS, the automatons detect the sediment-water interface by means of porosity-based rules and, having encountered the water column, do not venture any farther upwards. Any resuspended particles subsequently fall downwards onto the sediment surface. Thus, the sediment-water interface restricts the movement of animals and, as a result, the displacement of particles. Clearly then, the step length PDF is skewed by such a boundary, which alters its mean and variance and, accordingly, the mixing parameters as described by Eqs. 5 and 6. This is evident in the depth profiles of the particle-tracking mixing parameters, $D_{b}^{p}$ and $v_{b}^{p}$, calculated after 5 years to guarantee the PDFs are well-defined (Fig. 2). Both parameters are relatively constant with depth, but exhibit boundary effects at both the sediment-water interface and at the base of the mixed layer. $D_{b}^{p}$ can be seen to increase toward the boundaries before decreasing rapidly, while $v_{b}^{p}$ increases to a maximum at the upper-bound and decreases to a minimum at the lower-bound. Note that the decline of $D_{b}^{p}$ toward the surface does not mean a decline in mixing, but represents a change in the how mixing is manifested, as it is accompanied by an increase in $v_{b}^{p}$. The observed advective drift at the boundaries is inevitable, as particle transport is impeded in one direction creating a directional bias. In nature, the boundary effect at the base of the mixed layer is likely to be far less pronounced — if at all present—-since a natural mixed layer is not rigidly defined as it is in LABS. In contrast, the sediment-water interface constitutes a definite boundary to infauna and will therefore generate an advective boundary effect—although it may not necessarily be obvious. The observed boundary effect is similar in appearance to spatially variable compaction. In the case of radioisotope tracers, advection as a decreasing function of depth appears as a "flattening" of the tracer profile and is, therefore, not especially apparent (Christensen, 1982). For transient tracers, the effect will not be observable if the profile resolution is greater than the extent of the boundary layer. Figure $2 b$ shows that advection 


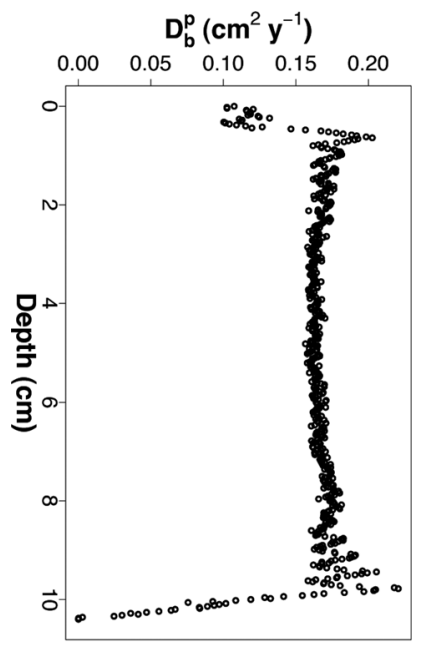

(a)

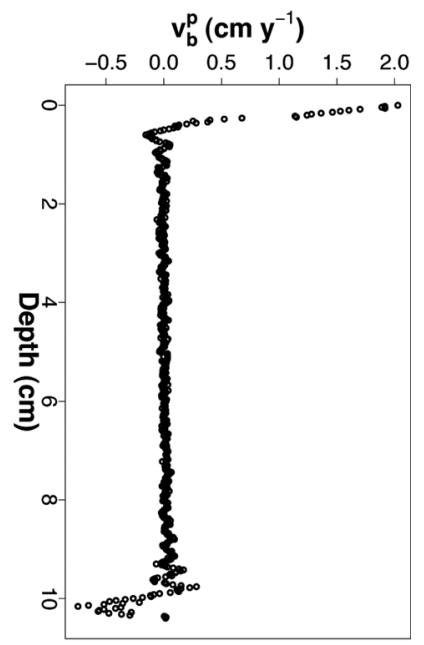

(b)

Figure 2. Particle-tracking mixing parameters (a) $D_{b}^{p}$ and (b) $v_{b}^{p}$ versus depth.

decreases to a negligible level away from the boundaries; nevertheless, introduced particles encounter the boundary effect immediately, as the tracer is input at the sediment-water interface. Therefore, the change in the nature of mixing observed when using transient tracers does not necessarily represent a change in mixing mechanism, but can be brought about by constraints imposed on bioturbation by the sediment-water interface.

\section{b. Tracer-derived mixing estimates}

Mixing parameters are estimated from tracer distributions, such as Figure 1, by applying the biodiffusion model, as outlined earlier. Output tracer profiles were sectioned into $5 \mathrm{~mm}$ slices and Eq. 4 was fit to these profiles using a least-squares cost function minimized by means of the Broyden-Fletcher-Goldfarb-Shanno method, as implemented in R (R Development Core Team, 2006). These model fits furnish values for the mixing parameters $D_{b}^{t}$ and $v_{b}^{t}$. The simulation outlined above was run 10 times and the average values of $D_{b}^{t}$ and $v_{b}^{t}$ are plotted against time in Figure 3. For reference, the depth-averaged values of $D_{b}^{p}$ and $v_{b}^{p}$ are also plotted in this figure (dashed lines). As shown later in Section $4 \mathrm{~d}$, these particle-tracking parameters are invariable in time. Immediately obvious in Figure 3a is that $D_{b}^{t}$ is a decreasing function of time, tending to an asymptote at $D_{b}^{p}$ in the long time limit. Similarly, Figure $3 \mathrm{~b}$ shows that $v_{b}^{t}$ initially overestimates the average bioadvection tending to an asymptote with time. Also plotted in Figure 3 are error bars representing the standard error of the mean. The variability in both $D_{b}^{t}$ and $v_{b}^{t}$ is only apparent in the first two or three points of the plots and even then is negligibly small. These simulations are in agreement 


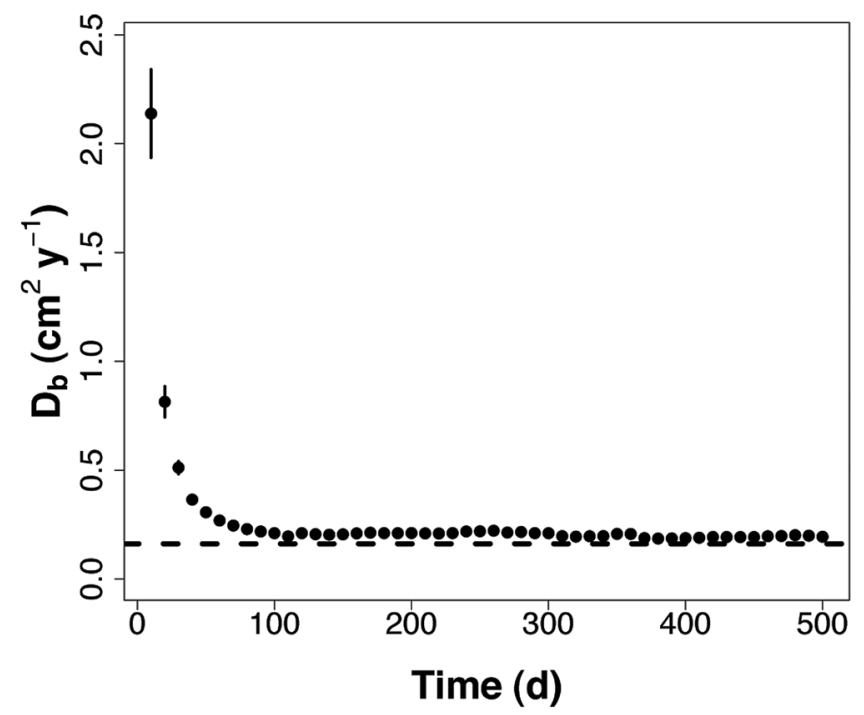

(a)

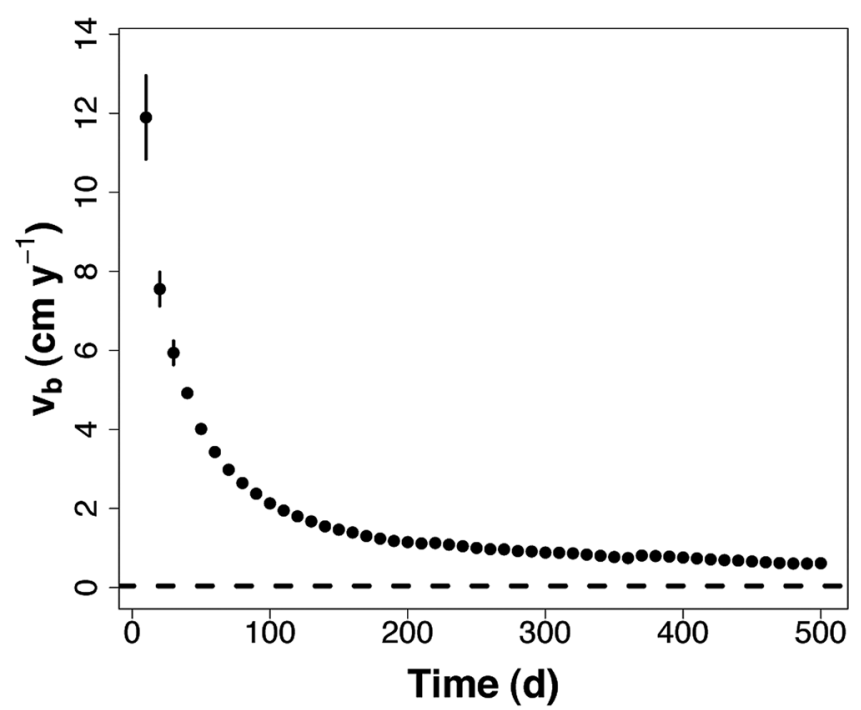

(b)

Figure 3. Tracer-derived mixing parameters (a) $D_{b}^{t}$ and (b) $v_{b}^{t}$ versus time with the tracer introduced at the sediment-water interface. The points represent the average of 10 simulations, while the error bars represent the standard error of the mean. The dashed line represents the depth-averaged value of (a) $D_{b}^{p}$ and (b) $v_{b}^{p}$. 
with field observations in that the biodiffusive coefficient is a decreasing function of time despite indiscriminate mixing of particles. The root of this trend is examined below.

To examine mixing of transient tracers in the absence of boundary effects, simulations using the previous parameter set were undertaken with the pulse of tracer introduced in the center of the mixed layer, instead of at the sediment-water interface. As advection is negligible away from the boundaries, the profile maximum remained stationary and mixing is modeled solely as diffusion. Furthermore, as the tracer is unaware of the boundaries for short times, the model solution can be simplified to that of diffusion on an infinite domain (Carslaw and Jaeger, 1965):

$$
C(x, t)=\frac{1}{\sqrt{4 \pi D_{b}^{t} t}} e^{\left(\frac{-x^{2}}{4 D_{b}^{t}}\right)}
$$

Fitting Eq. 7 to tracer profiles provides values for $D_{b}^{t}$. Once again, the simulation was run 10 times and the average values of $D_{b}^{t}$ are plotted against time in Figure 4. The figure shows the same trend as observed previously, thus the trend persists when the tracer is uninfluenced by boundary effects. Also plotted in Figure 4 is $N_{t}$, the average number of mixing events experienced by a particle, defined as $N_{t}=\frac{t}{\tau}$. As in Figure $3, D_{b}^{t}$ appears as a decreasing function of time, significantly overestimating mixing in the short-term, that is, when $N_{t}$ is small. The reason for this lies in the scales of observation and mixing. The characteristic

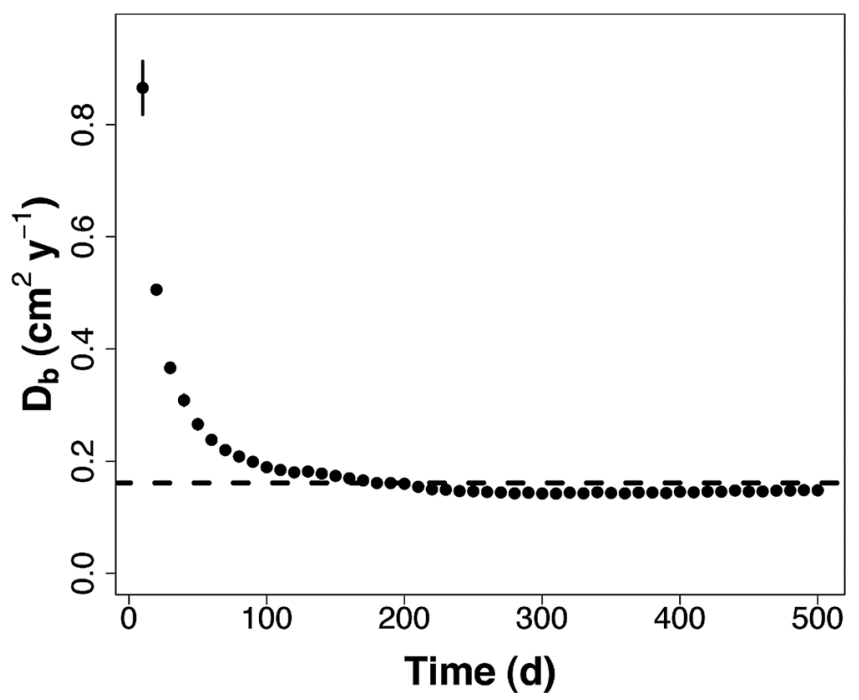

Figure 4. Tracer-derived mixing coefficient $D_{b}^{t}$ versus time with the tracer introduced in the center of the mixed layer. The points represent the average of 10 simulations, while the error bars represent the standard error of the mean. The dashed line represents the depth-averaged value of $D_{b}^{p}$. 
length-scale of a tracer profile, $x_{t}$, is given by the root of the variance,

$$
x_{t}=\sqrt{2 D_{b}^{t} t}
$$

Figure 4 shows that after a sufficiently long period of time $D_{b}^{t}$ and $D_{b}^{p}$ are in agreement and, accordingly,

$$
x_{t}=\sqrt{2 D_{b}^{t} t}=\sqrt{2 D_{b}^{p} t}=\sqrt{\frac{\sigma^{2} t}{\tau}}=\sigma \sqrt{N_{t}}
$$

Eq. 9 suggests that for a small number of mixing events the tracer length-scale, $x_{t}$, is comparable in magnitude to the mixing length, $\sigma$. However, modeling theory asserts that for the diffusion analogy to be valid, the scale of mixing must be much smaller than the scale of observation, that is, $x_{t} \gg \sigma$ (Boudreau, 1986a; Meysman et al., 2003). Clearly, this criterion is violated for short time-scales. In reality, mixing ensures that the tracer lengthscale is always larger than the mixing length and, as a result, the diffusion analogy and Eq. 9 break down, resulting in erroneous values for $D_{b}^{t}$. Unfortunately, failure of the model is not apparent from the tracer profiles. This is seen in Figure 5, which shows model fits to profiles from the initial simulation. Despite violation of the model's criteria and the influence of boundary effects, the biodiffusion model still provides sterling fits to tracer distributions. The profiles give no hint that the model is invalid and, therefore, the time scale of mixing needs to be inferred by calculating reworking rates in order to assess the validity of the model in practice. While the preceding discussion focuses upon the constant coefficient biodiffusion

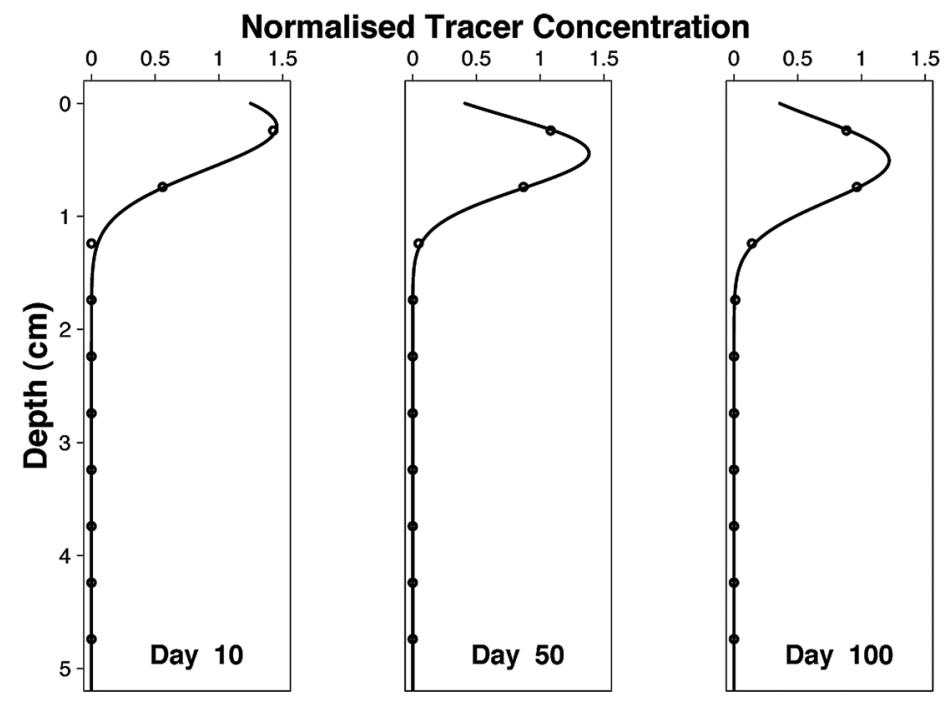

Figure 5. Three examples of biodiffusion model fits to LABS generated profiles when the model criteria are invalid. 
model, the principle extends to its spatially variable counterpart (e.g., an exponentially decreasing $D_{b}$ ), as such models are derived from the same assumptions.

These results demonstrate $D_{b}^{t}$ as a decreasing function of time, in agreement with field studies (Fornes et al., 2001; Smith et al., 2001). However, our simulations show that preferential mixing need not be invoked to produce such a trend, but rather that a temporal trend in the biodiffusion coefficient occurs inherently when the biodiffusion model is applied over short time scales.

\section{c. Acclimation period}

The time taken for the tracer-derived mixing coefficient to become representative of the actual mixing rate is a function of rest period: the more frequently particles are mixed, the faster $D_{b}^{t}$ tends to $D_{b}^{p}$. To demonstrate this, further model runs were undertaken with exactly the same parameters as previously used, except for faunal density. Two different populations were used with faunal densities of 1,000 and 10,000 individuals $\mathrm{m}^{-2}$ to represent modest and intensive mixing regimes, respectively. As with the previous simulation, the tracer pulse was introduced in the center of the mixed layer and $D_{b}^{t}$ values were determined by fitting Eq. 7 to tracer profiles sectioned into $5 \mathrm{~mm}$ layers. To allow the two simulations to be compared, $D_{b}^{t}$ data were normalized using the relevant depth-averaged value of $D_{b}^{p}$, such that both data sets tend to an asymptote at 1 . As the variation between identical simulations is relatively small (e.g., Figs. 3 and 4), only one simulation was undertaken for each scenario considered here. Figure 6 shows both data sets versus time over a period of 500 days, providing an appreciation of the error incurred by using the biodiffusion on short timescales. The tracer-derived mixing coefficient for the modest mixing regime (e.g., a deep-sea environment) is almost $3000 \%$ of the actual mixing rate after 10 days. Even the intensive mixing regime (e.g., a coastal environment) has a tracer-derived mixing coefficient of over $300 \%$ the actual mixing rate after 10 days. In both cases, the error decreases as time, and the number of mixing events, increase. Clearly, the intensive mixing regime tends to the asymptote more rapidly than the modest mixing regime, coming to within $10 \%$ of the correct mixing coefficient after 110 days, while the modest mixing regime required 790 days. These times suggest, as a rule of thumb, on average at least 9 mixing events are needed for tracerderived mixing coefficient to be within $10 \%$ of actual mixing rate. When the two data sets are plotted against $N_{t}$, as opposed to time, they are coincident demonstrating the error in tracer-derived mixing coefficient as a function of average number of mixing events (Fig. 7).

From the point of view of diagenetic models, the tolerable error in $D_{b}$ is determined by means of a sensitivity analysis and will vary markedly depending on the system considered. Perhaps more importantly, however, are the implications of these results with regard to using $D_{b}$ as a comparative metric. As discussed in detail above, misapplication of the biodiffusion model can invent trends in bioturbation intensity that can be misconstrued as differential mixing; conversely, actual trends in $D_{b}$ can also be attenuated. By way of an example, consider the empirical relationship between water depth and biodiffusion coefficient: biological 


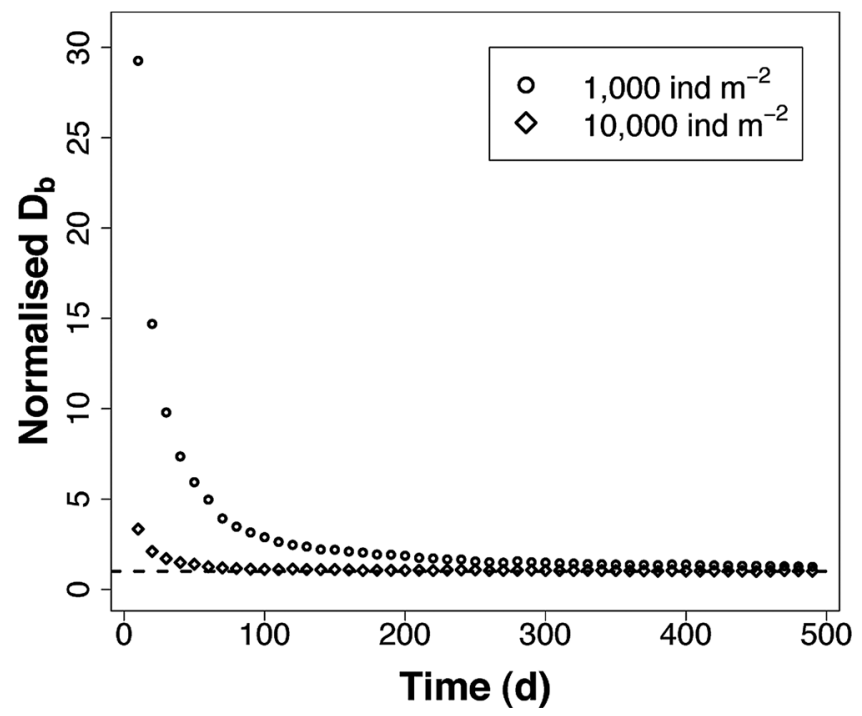

Figure 6. Tracer-derived mixing coefficient $D_{b}^{t}$ versus time for two different faunal densities. The tracer is introduced in the center of the mixed layer. For the sake of comparison, each data set is normalized using the appropriate depth-averaged value of $D_{b}^{p}$.

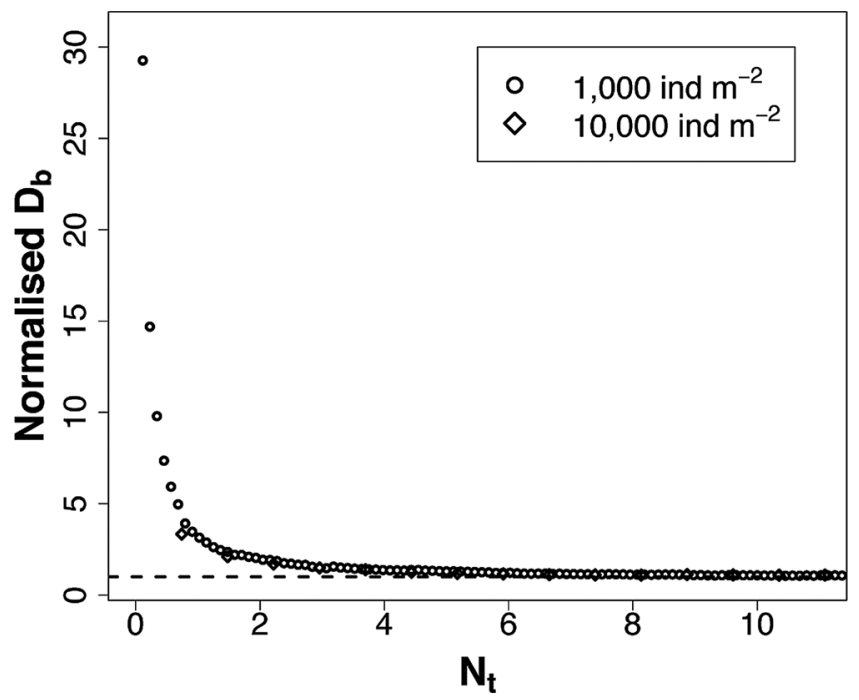

Figure 7. Tracer-derived mixing coefficient $D_{b}^{t}$ versus $N_{t}$ for two different faunal densities. The tracer is introduced in the center of the mixed layer. For the sake of comparison, each data set is normalized using the appropriate depth-averaged value of $D_{b}^{p}$. 
mixing rate decreases as water depth increases (Boudreau, 1994). In shallower sediments, particles are displaced more frequently than deep-sea sediments meaning that the biodiffusion analogy is more likely appropriate. Thus, deep-sea sediments are mixed to a lesser degree, but the biodiffusion model is more likely to yield erroneous overestimates of mixing intensity in these settings. The potential upshot is that the relationship between water depth and $D_{b}$ is mischaracterized and that bioturbation intensity may actually decrease more rapidly with water depth than previously thought.

\section{d. Particle-tracking over time}

In LABS, each and every particle can be tracked individually throughout simulations and, thus, mixing can be examined as a function of time. This is necessary to establish that the temporal trend in tracer-derived mixing coefficients is not simply a model artifact due to transient effects.

In his seminal work on Brownian motion, Einstein (1905) showed that the mean square displacement of a large number of particles subject to diffusion is a linear function of time. Specifically,

$$
\left\langle x^{2}\right\rangle=2 D t
$$

where $\left\langle x^{2}\right\rangle$ denotes the mean square displacement of particles, $D$ represents the diffusion coefficient, and $t$ is time. The derivation of this formula assumes a symmetric step length PDF and, thus, no advection is present. When particle displacements are anisotropic, the resulting drift must be accounted for by using the variance of the step length distribution, as opposed to the mean square displacement. For a symmetric step length PDF, the variance is equal to the mean square displacement, as the mean displacement is zero.

To calculate the mean square displacement of particles in LABS, the following equation is used:

$$
\left\langle x^{2}\right\rangle=\frac{1}{p} \sum_{i=1}^{p} \Delta x_{i}^{2}
$$

where $\Delta x_{i}$ denotes the displacement of particle $i$ from its initial location and $p$ is the number of particles in the system. Similarly, the mean displacement of particles, $\langle x\rangle$, is determined using,

$$
\langle x\rangle=\frac{1}{p} \sum_{i=1}^{p} \Delta x_{i}
$$

From these values, the variance of particle displacements is calculated:

$$
\sigma_{p}^{2}=\left\langle x^{2}\right\rangle-\langle x\rangle^{2}
$$

This approach can be used to examine mixing in both the vertical and lateral aspects. (Note that the subscript $p$ differentiates the variance of particle displacements from the variance of the step length distribution.) For a simulation using the original parameter set, a plot of the 


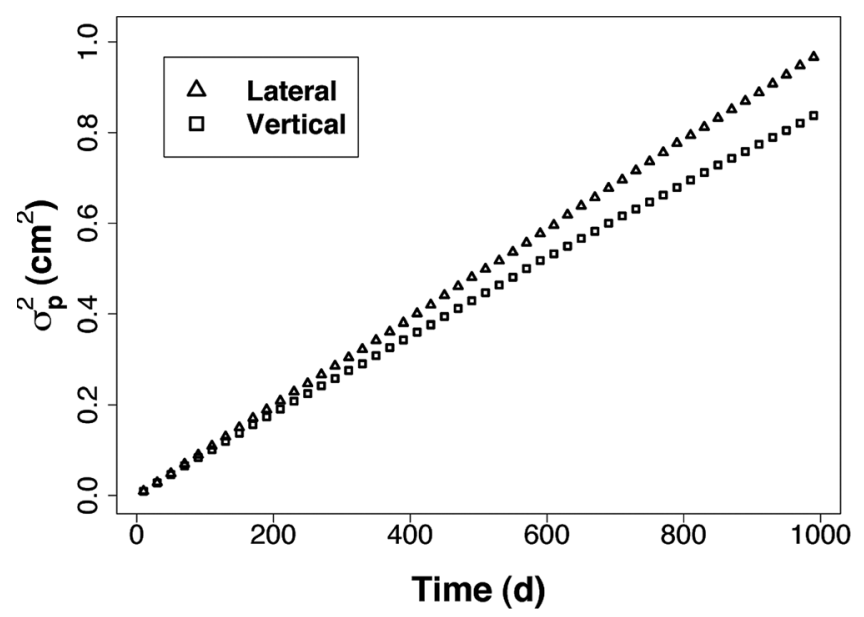

Figure 8. The variance of particle displacements versus time for the original parameter set.

variance of particle displacements against time reveals a near linear relationship, suggesting that mixing is temporally invariable (Fig. 8). Boudreau et al. (2001) previously reported that mixing in LABS is initially subdiffusive (i.e., the variance of particle displacements is sublinear function of time), becoming diffusive with time. However, the original FORTRAN version of LABS contained an error in the calculation related to the wrapping of particles across the left- and right-hand boundaries. This error is corrected in the current version of the model.

In Figure 8, mixing initially appears isotropic, but vertical mixing appears to decline slightly with time. A further model run was undertaken with the same parameters except for the length of the automatons and their gut-to-body ratio, which were set at $5 \mathrm{~cm}$ and 0.5 , respectively. The rationale was to increase the gut size and, therefore, the retention time of particles in the gut, so particles are transported over larger distances. The variance of displacements for this simulation versus time is plotted in Figure 9. This figure shows the variance of vertical particle displacements tending to an asymptote with time. Interpreting these results with Eq. 10 in mind, one may deduce, erroneously, that vertical mixing intensity decreases with time. But use of Eq. 10 is inappropriate in this case, as it is derived for an infinite domain. In LABS, horizontal transport is unconstrained, as particles can wrap across the left- and right-hand boundaries indefinitely; however, vertical transport is limited by the presence of the sediment-water interface and the base of the mixed layer. Accordingly, there is a maximal vertical distance a particle can stray from its initial position, giving rise to the noted asymptote. The position of the asymptote can be derived by considering the distribution and movement of particles.

Let $P(x, t)$ be the normalized vertical distribution of particles at time, $t$. Initially, particles are uniformly distributed throughout the mixed layer and, therefore, $P(x, 0)=\frac{1}{L}$, where $L$ 


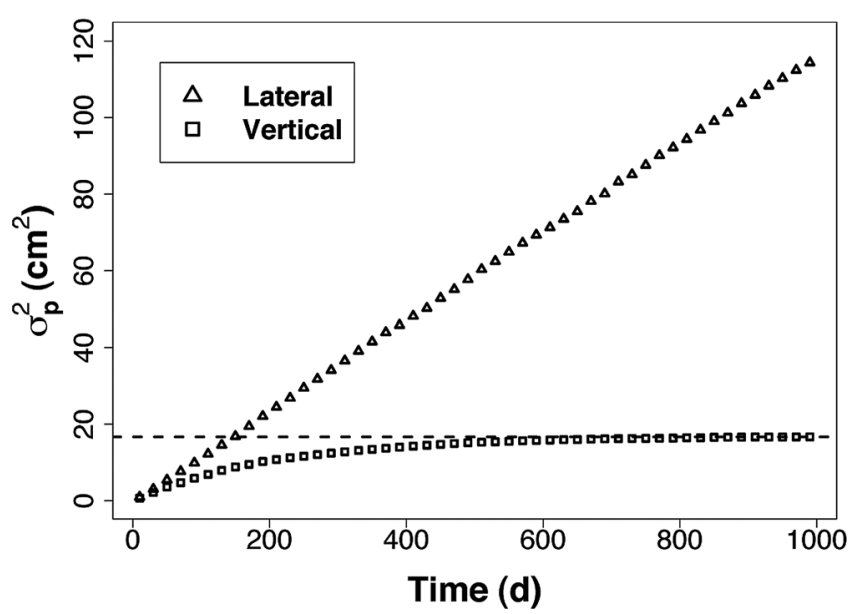

Figure 9. The variance of particle displacements versus time for the original parameter set, but with larger organisms (5 cm in length) and larger gut-to-body ratios $(0.5)$. The dashed line represents $\frac{L^{2}}{6}$, where $L$ is the mixed layer depth.

is the mixed layer depth. Assuming the particles move diffusively in an insulated domain, their distribution is governed by the equation,

$$
\frac{\partial P}{\partial t}=D \frac{\partial^{2} P}{\partial x^{2}}
$$

The initial condition forms a steady-state solution to this equation, therefore, $P(x)=\frac{1}{L}$. In the simulations presented here, anisotropic transport occurs only near the boundaries, which constitutes but a small portion of the mixed layer. As a result, $\langle x\rangle$ is negligible in Eq. 13 and the variance effectively becomes the mean square displacement. This allows the following argument to be simplified. The mean square displacement for particles originally at point $x_{0}$ is,

$$
\left\langle x^{2}\right\rangle_{s}=\int_{0}^{L}\left(x-x_{0}\right)^{2} P(x) d x=\frac{1}{L} \int_{0}^{L}\left(x-x_{0}\right)^{2} d x=\frac{L^{2}}{3}-L x_{0}+x_{0}^{2}
$$

where the subscript $s$ denotes a point source. However, the particles do not all begin at the same point; therefore, the distribution of initial positions, $P\left(x_{0}\right)$, must be accounted for:

$$
\left\langle x^{2}\right\rangle=\int_{0}^{L}\left\langle x^{2}\right\rangle_{s} P\left(x_{0}\right) d x_{0}=\frac{1}{L} \int_{0}^{L}\left(\frac{L^{2}}{3}-L x_{0}+x_{0}^{2}\right) d x_{0}=\frac{L^{2}}{6}
$$

This result suggests that the vertical mean square displacement, and therefore the variance of vertical particle displacements, should tend to $\frac{L^{2}}{6}$ with time. Plotting this value in Figure 9 (dashed line) reveals that this is indeed the observed asymptote. 


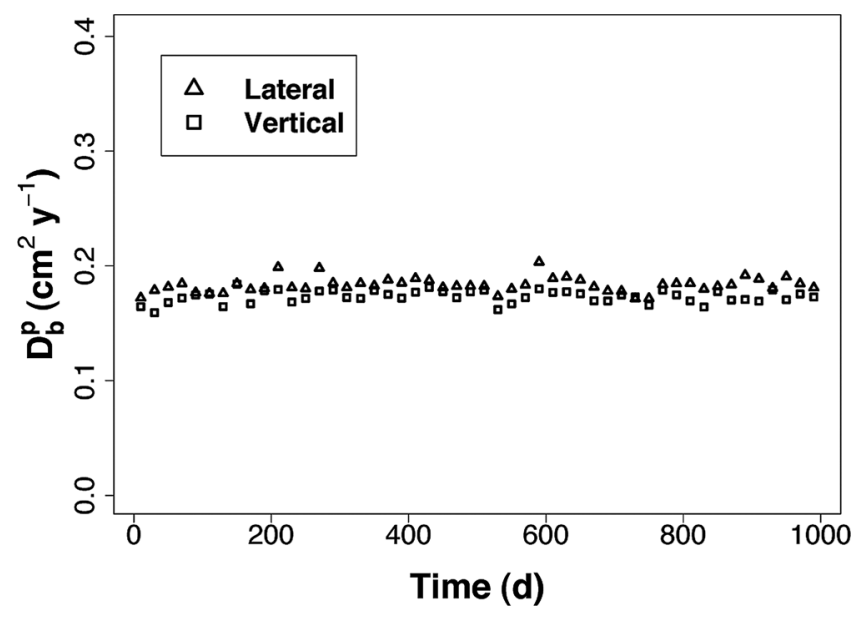

Figure 10. The biodiffusion coefficient versus time as determined from the variance of particle displacements over 10 day intervals for a simulation with the original parameter set.

Owing to the influence of boundaries, analysis of the variance proves ineffective in the vertical aspect over long periods of time. However, as seen in Figure 8, the variance of the vertical displacements appears linear in time and, therefore, unaffected by boundaries over a sufficiently short period of time. This can be taken advantage of by dividing time into a series of short intervals and measuring mixing over each interval for comparison. As with the previous approach, the variance of particle displacements is used, calculated from particles' current and initial positions. The vital difference is that after each calculation the particles' current positions are set as their initial positions, effectively resetting particle tracking. The result is a measure of the variance of particle displacements for each interval independent of all other intervals and unaffected by boundaries. Dividing this value by twice the length of the interval provides a mixing rate (see Eq. 10), allowing the temporal variability of mixing to be examined. This is shown in Figure 10 for a model run with the original parameter set and an interval of 10 days. Horizontal and vertical mixing rates are in close agreement with each other as well as the depth-averaged $D_{b}^{p}$ value, and are relatively constant in time. While there is some variability due to the stochastic nature of the model, there is no temporal trend in mixing for either direction. This confirms that the observed trend in tracer-derived mixing coefficients is not a product of transient effects related to the initialisation of the model, but is a genuine phenomenon.

\section{Conclusions}

The biodiffusion model is the most widely used quantitative description of bioturbation. Frequently, this model is used with conservative, transient tracers, such as luminophores or other introduced particles, to study biogenic mixing over short time scales. Past studies have 
shown mixing intensity, as quantified by the biodiffusion model, declines with time; mixing also progresses from bioadvective or nonlocal transport to diffusive mixing (Fornes et al., 2001; Smith et al., 2001). These observations have been previously explained by so-called "age-dependent" mixing, a preferential mixing mechanism. Nonetheless, modeling work presented here demonstrates that these field observations are to be expected in the absence of any particle-selectivity. LABS simulations reveal that applying the biodiffusion model in the short-term invents a temporal trend in the mixing rate, even when mixing is constant and indiscriminate. The error in the tracer-derived mixing coefficient can be considerable and only approaches the actual mixing rate after sufficient mixing events have been experienced. Results suggest, as a rule of thumb, on average at least 9 mixing events are needed before the biodiffusion model becomes valid. Furthermore, simulations presented here demonstrate a shift from advective to diffusive mixing, as observed in nature, resulting from boundary effects. The constraints imposed on bioturbation near boundaries creates a directional bias and, consequently, an advective drift. Beyond this boundary layer, mixing can progress diffusively.

These results add weight to the argument against using the biodiffusion model on short time-scales and impetus to the search for a suitable short-term description of bioturbation. Work presented here, when coupled with previous studies (i.e., Reed et al., 2006), suggests that mixing is yet to be parameterized accurately on short time scales. This is a significant issue when considering short-term diagenetic processes, such as the fate of labile organic matter.

Acknowledgments. We thank Drs. Bob Aller and Pierre Regnier, plus two anonymous reviewers for their helpful comments on the manuscript. We also wish to thank the US Office of Naval Research, and Program Manager, Dr. Jim Eckman, for generous support for this project (N00014-02-1-0107).

\section{REFERENCES}

Aller, R. C. 1982. The effects of macrobenthos on chemical properties of marine sediment and overlying water, in Animal-Sediment Interactions, P. L. McCall and M. J. S. Tevesz, eds., Plenum Publishing Corporation.

1988. Benthic fauna and biogeochemical processes in marine sediments: the role of burrow structures, in Nitrogen Cycling in Coastal Marine Sediments, T. H. Blackburn and J. Sørensen, eds., John Wiley \& Sons Ltd.

Benninger, L. K., R. C. Aller, J. K. Cochran and K. K. Turekian. 1979. Effects of biological sediment mixing on the ${ }^{210} \mathrm{~Pb}$ chronology and tracer metal distribution in a Long Island Sound sediment core. Earth. Planet. Sci. Lett., 43, 241-259.

Berger, W. H. and G. R. Heath. 1968. Vertical mixing in pelagic sediments. J. Mar. Res., 26, 134-143.

Berner, R. A. 1980. Early Diagenesis: A Theoretical Approach, Princeton University Press.

Bosworth, W. S. and L. J. Thibodeaux. 1990. Bioturbation: A facilitator of contaminant transport in bed sediment. Environ. Prog., 9, 211-217.

Boudreau, B. P. 1986a. Mathematics of tracer mixing in sediments: 1. Spatially-dependent, diffusive mixing. Am. J. Sci., 286, 161-198.

- 1986b. Mathematics of tracer mixing in sediments: 2. Nonlocal mixing and biological conveyor-belt phenomena. Am. J. Sci., 286, 199-238. 
1989. The diffusion and telegraph equations in diagenetic modelling. Geochim. Cosmochim. Acta, 53, 1857-1866.

1994. Is burial velocity a master parameter of bioturbation? Geochim. Cosmochim. Acta, 58, 1243-1249.

Boudreau, B. P., J. Choi, Filip Meysman and F. François-Carcaillet. 2001. Diffusion in a latticeautomaton model of bioturbation by small deposit-feeders. J. Mar. Res., 59, 749-768.

Boudreau, B. P. and D. M. Imboden. 1987. Mathematics of tracer mixing in sediments: 3. The theory of nonlocal mixing within sediments. Am. J. Sci., 287, 693-719.

Caron, A., G. Derosiers, C. Retière and S. Brenot. 1996. The behaviour of Nephtys caeca inside sediment under experimental conditions. Comptes rendus de l'academie des sciences: Sciences de la vie, 319, 417-423.

Carslaw, H. S. and J. C. Jaeger. 1965. Conduction of Heat in Solids (2nd edition), Oxford (Clarendon Press).

Choi, J., F. François-Carcaillet and B. P. Boudreau. 2002. Lattice-automaton bioturbation simulator (LABS): implementation for small deposit feeders. Comput. Geosci., 28, 213-222.

Christensen, E. R. 1982. A model for radionuclides in sediments influenced by mixing and compaction. J. Geophys. Res., 87, 566-572.

Cochran, J. K. 1985. Particle mixing rates in sediments of the eastern equatorial Pacific: Evidence from ${ }^{210} \mathrm{~Pb},{ }^{239,240} \mathrm{Pu}$ and ${ }^{137} \mathrm{Cs}$ distributions at MANOP sites. Geochim. Cosmochim. Acta, 49, 1195-1210.

DeMaster, D. J., B. A. McKee, C. A. Nittrouer, D. C. Brewster and P. E. Biscaye. 1985. Rates of sediment reworking at the HEBBLE site based on measurements of Th-234, Cs-137 and Pb-210. Mar. Geol., 66, 133-148.

Dorgan, K. M., P. A. Jumars, B. Johnson, B. P. Boudreau and E. Landis. 2005. Burrow extension by crack propagation. Nature, $433,475$.

Einstein, A. 1905. Über die von der molekularkinetischen Theorie der Wärme gefordete Bewegung von in ruhenden Flssigkeiten suspendierten Teilchen. Annalen der Physik, 17, 549-560.

Fisher, J. B., W. L. Lick, P. L. McCall and J. A. Robbins. 1980. Vertical mixing of lake sediments by tubificid oligochaetes. J. Geophys. Res., 85, 3997-4006.

Forbes, T. L. 1984. Aspects of the feeding biology of Capitella capitata Species 1: Measurements of ingestion selectivity, egestion rate, and absorption efficiency, Master's thesis, SUNY at Stoney Brook.

Fornes, W. L., D. J. DeMaster and C. R. Smith. 2001. A particle introduction experiment in Santa Catalina Basin sediments: Testing the age-dependent mixing hypothesis. J. Mar. Res., 59, 97-112.

François, F., J.-C. Poggiale, J.-P. Durbec and G. Stora. 1997. A new approach for the modelling of sediment reworking induced by a macrobenthic community. Acta Biotheor., 45, 295-319.

Gerino, M., R. C. Aller, C. Lee, J. K. Cochran, J. Y. Aller, M. A. Green and D. Hirschberg. 1998. Comparison of different tracers and methods used to quantify bioturbation during a spring bloom: 234-thorium, luminophores and chlorophyll a. Estuar. Coast. Shelf Sci., 46, 531-547.

Gerino, M., G. Stora and J.-P. Durbec. 1994. Quantitative estimation of biodiffusive and bioadvective sediment mixing: In situ experimental approach. Oceanol. Acta, 17, 547-554.

Goldberg, E. D. and M. Koide. 1962. Geochronological studies of deep sea sediments by the ionium/thorium method. Geochim. Cosmochim. Acta, 26, 417-450.

Goreau, T. J. 1977. Quantitative effects of sediment mixing on stratigraphy and biogeochemistry: a signal theory approach. Nature, 265, 525-526.

Grassle, J. F. and J. P. Grassle. 1974. Opportunistic life histories and genetic systems in marine benthic polychaetes. J. Mar. Res., 32, 253-284. 
Grassle, J. P. and J. F. Grassle. 1976. Sibling species in the marine pollutant indicator Capitella (Polychaeta). Science, 192, 567-569.

Guinasso, N. L. and D. R. Schink. 1975. Quantitative estimates of biological mixing rates in abyssal sediments. J. Geophys. Res., 80, 3032-3043.

Hughes, B. D. 1995. Random walks and random environments. Volume 1: Random Walks, Clarendon Press.

Johnson, B. D., B. P. Boudreau, B. S. Gardiner and R. Maass. 2002. Mechanical response of sediments to bubble growth. Mar. Geol., 187, 347-363.

Jumars, P. A., L. M. Mayers, J. W. Deming, J. A. Baross and R. A. Wheatcroft. 1990. Deep-Sea deposit-feeding strategies suggested by environmental and feeding constraints. Philos. Trans. R. Soc. London, 331, 85-101.

Lopez, G. and J. S. Levinton. 1987. Ecology of deposit-feeding animals in marine sediments. Q. Rev. Biol., 62, 235-260.

Méndez, N., I. Linke-Gamenick, V. E. Forbes and D. J. Baird. 2001. Sediment processing in Capitella spp. (Polychaeta: Capitellidae): strain-specific differences and effects of the organic toxicant fluoranthene. Mar. Biol., 138, 311-319.

Meysman, F. J. R., B. P. Boudreau and J. J. Middelburg. 2003. Relations between local, nonlocal, discrete and continuous models of bioturbation. J. Mar. Res., 61, 391-410.

Officer, C. B. and D. R. Lynch. 1982. Interpretation procedures for the determination of sediment parameters from time-dependent flux inputs. Earth. Planet. Sci. Lett., 61, 55-62.

Penry, D. L. and P. A. Jumars. 1990. Gut architecture, digestive constraints and feeding ecology of deposit-feeding and carnivorous polychaetes. Oecologia, 82, 1-11.

R Development Core Team. 2006. R: A Language and Environment for Statistical Computing, R Foundation for Statistical Computing, Vienna, Austria, ISBN 3-900051-07-0.

Reed, D. C., K Huang, B. P. Boudreau and F. J. R. Meysman. 2006. Steady-state tracer dynamics in a lattice-automaton model of bioturbation. Geochim. Cosmochim. Acta, 70, 5855-5867.

Rhoads, D. C. 1974. Organism-sediment relations on the muddy sea floor. Oceanogr. Mar. Biol., 12, 263-300.

Rice, D. L. 1986. Early diagenesis in bioadvective sediments: relationships between the diagenesis of beryllium-7, sediment reworking rates and the abundance of conveyor-belt deposit feeders. J. Mar. Res., 44, 149-184.

Shull, D. H. 2001. Transition-matrix model of bioturbation and radionuclide diagenesis. Limnol. Oceanogr., 46, 905-916.

Smith, C. R., W. Berelson, D. J. DeMaster, F. C. Dobbs, D. Hammond, D. J. Hoover, R. H. Pope and M. Stephens. 1997. Latitudinal variations in benthic processes in the abyssal equatorial Pacific: control by biogenic particle flux. Deep-Sea Res. II, 44, 2295-2317.

Smith, C. R., D. J. DeMaster and W. L. Fornes. 2001. Mechanisms of age-dependent mixing on the bathyal California margin: the young and the restless., in Organism-Sediment Interactions, J. Y. Aller, S. A. Woodin and R. C. Aller, eds., University of South Carolina Press.

Smith, C. R., R. H. Pope, D. J. DeMaster and L. Magaard. 1993. Age-dependent mixing of deep-sea sediments. Geochim. Cosmochim. Acta, 57, 1473-1488.

Solan, M., B. D. Wigham, I. R. Hudson, C. H. Coulon, R. Kennedy, K. Norling, H. Nilsson, and R. Rosenberg. 2004. In situ quantification of infaunal bioturbation using fluorescent sediment profile imaging (f-SPI), luminophore tracers and model simulation. Mar. Ecol-Prog Ser., 271, 1-12.

Tedesco, L. P. and H. R. Wanless. 1991. Generation of sedimentary fabrics and facies by repetitive excavation and storm infilling of burrow networks, Holocene of South Florida and Caicos Platform, B. W. I. Palaios, 6, 326-343. 
Turnewitsch, R., U. Witte and G. Graf. 2000. Bioturbation in the abyssal Arabian Sea: influence of fauna and food supply. Deep-Sea Res. II, 47, 2877-2911.

Wheatcroft, R. A. 1991. Conservative tracer study of horizontal sediment mixing rates in a bathyal basin, California borderland. J. Mar. Res., 49, 565-588.

1992. Experimental tests for particle size-dependent bioturbation in the deep ocean. Limnol. Oceanogr., 37, 90-104.

Wheatcroft, R. A., P. A. Jumars, C. R. Smith and A. R. M. Nowell. 1990. A mechanistic view of the particulate biodiffusion coefficient: step lengths, rest periods and transport directions. J. Mar. Res., 48, 177-207.

Received: 2 January, 2007; revised: 18 December, 2007. 